\title{
Prinzipien einer deutsch-tschechischen Teamentwicklung
}

\author{
Christian Eberhardt
}

Die allgemein gängigen Modelle der Gruppenentwicklung gehen davon aus, daß jede Gruppe im Laufe ihres Lebenszyklus klar von einander abgrenzbare Phasen durchläuft (vgl. Tuckman, 1995). Neuere Untersuchungen weisen jedoch darauf hin, daß die realiter beobachtbare Entwicklung von Arbeitsgruppen nicht der Abfolge unitärer Teambildungsphasen folgt. So kommt Gersick (1988; vgl. 1990) in einer qualitativen Analyse von Projektgruppen zu dem Schluß, daß Arbeitsgruppen nicht erst eine forming, storming und norming-Phase durchleben müssen, bis sie sich der produktiven Zusammenarbeit zuwenden können; vielmehr wird bereits im ersten Zusammentreffen der Arbeitsgruppe der Rahmen der gemeinsamen Problembewältigung festgelegt und ein Spektrum nachhaltig wirksamer Verhaltensroutinen ausgeformt. Dem Grundgedanken dieses Modells entsprechend ist die Gruppenentwicklung durch verschiedene Perioden der Stabilität in den Verhaltensmustern der Gruppenmitglieder charakterisiert: nach einer Periode der Stabilität in Folge der nachhaltigen Erstkontakterfahrungen setzen erst zur Halbzeit der Projektarbeit Veränderungsprozesse ein, führen unter Umständen zur Revision bis dato gültiger Verhaltensroutinen und münden anschließend in einer erneuten Periode der Stabilität.

Wendet man diese Untersuchungsergebnisse Gersicks (1988) auf das Feld der bi-kulturellen Teamentwicklung an, so liegt die Schlußfolgerung nahe, daß sowohl der Einstieg in die bi-kulturelle Zusammenarbeit als auch die Halbzeit der Projektarbeit als kritische Phasen im Mittelpunkt von Interventionsmaßnahmen stehen müssen. Besonders virulent ist diese Aussage vor dem Hintergrund der besonderen Bedeutung des Erstkontaktes in der deutsch-tschechischen Begegnung (Eberhardt, 2000): gerade von tschechischer Seite aus verhärten sich im oft mit critical incidents gespickten erstmaligen Aufeinandertreffen deutscher und tschechischer Mitarbeiter durch die subjektive Bestätigung stereotyper Vorstellungen Verhaltensdispositionen, welche die künftige Zusammenarbeit erschweren und die immer wieder beobachtbare Konfliktspirale der tschechisch-deutschen Reaktanz-Dominanz-Dynamik in Gang setzen und schwelend wirksam halten.

Verpaßt eine Arbeitsgruppe den glücklichen 'Einstieg' in die Zusammenarbeit, so kann Gersicks (1988) Analyseergebnissen entsprechend weiters davon ausgegangen werden, da $\beta$ die in der ersten Periode der Projektarbeit gewonnenen suboptimalen Informationen und Erkenntnisse über die mit dem 
Arbeitsauftrag verbundene Problemstellung die Qualität der Problembearbeitung in der zweiten Hälfte der Projektzeit nachhaltig negativ beeinflussen. Empirischen Belegen Simons (2000) zufolge kann eine Gruppe, die sich in der ersten Periode ihrer Zusammenarbeit als nicht-produktiv erwies, dieses Defizit auch in der zweiten Periode nicht mehr aufholen; die Leistungsfähigkeit des Teams wäre in diesem Fall über die Zeit hinweg stabil - quasi auf einem niedrigen Niveau eingefroren. Fazit für die bi-kulturelle Teamentwicklung muß es deshalb sein, im Erstkontakt $\mathrm{zu}$ intervenieren: gegenseitige Anknüpfungspunkte zur Unterstützung von Vertrautheitserlebnissen und Sympathie müssen geschaffen und Anstöße zur gezielten Reflexion der Selbstund Fremdwahrnehmungen müssen gegeben werden. Ziel ist es, die sich in der Gruppe entwickelnden Interaktionsmuster so anzulegen, daß ein möglichst hoher Grad an Flexibilität und Selbstregulation hinsichtlich sich verändernder Arbeitsanforderungen auf der einen und sich verfestigenden, dysfunktionalen Verhaltensroutinen auf der anderen Seite gewährleistet wird.

Die qualitativen Ergebnisse Gersicks (1988), auf denen diese Schlußfolgerungen aufbauen, wurden von Simon (2000) - zumindest was die Relevanz des erstmaligen Zusammentreffens im Rahmen der Teamarbeit betrifft - auch anhand quantitativer Analyseergebnisse bestätigt; somit kann der Vorschlag, mit einer Teamentwicklungsmaßnahme gerade an der Supervision des Erstkontaktes deutscher und tschechischer Teamkollegen anzusetzen, als wohl fundiert angesehen werden.

Der zweite im Konzept Gersicks (1988) implizierte Vorschlag, auch die Halbzeit der Projektarbeit zur positiven Einflußnahme auf die Teamentwicklung zu nutzen, konnte zwar bis dato noch nicht empirisch untermauert werden, er kann jedoch aus Gründen der Plausibilität als gerechtfertigt gelten: die Ausführungen Simons (2000), welche sich mit der Problematik der Gruppeneffektivität und der Erfassung von Leistungspotentialen in Arbeitsgruppen befassen, bauen auf systemtheoretischen Grundannahmen auf; Gruppen werden als offene Systeme betrachtet, welche insbesondere durch das inhärente Prinzip der Äquifinalität charakterisiert sind (vgl. Watzlawick, 1996): die Leistungsfähigkeit eines Teams ist demnach nicht in erster Linie durch Input-Faktoren, sondern vielmehr durch die Natur des sozialen Prozesses - also das komplexe, überdauernde Interaktionsgeschehen innerhalb der Gruppe - determiniert.

Wird - wie Gersick (1988) feststellt - eine Arbeitsgruppe zur Halbzeit ihrer nur beschränkten zeitlichen Ressourcen gewahr und reflektiert daraufhin ihr bisheriges Vorgehen, so kann gerade diese kurze Phase der Selbstreflexivität dazu genutzt werden, gezielt auf die Interaktionskultur der Gruppe Einfluß zu nehmen: durch geschickte Moderation von Seiten eines Trainers/Supervisors ist die Evaluierung der bisherigen gemeinsamen Problemlösung anzuregen, darauf aufbauend die Explikation und Analyse gruppeninhärenter Schwachstellen voranzutreiben und die Unterstützung zur Entwicklung innovativer Wege der 
Zusammenarbeit im gemeinsamen Dialog zwischen Trainer und Teammitgliedern anzubieten.

Als konkreter Ansatz empfiehlt es sich, den zentralen Fragen der Prozeßanalyse nach Francis \& Young (1989) nachzugehen:

Welche Verhaltensweisen haben der Gruppe bei der bisherigen Aufgabenlösung geholfen?

Welche Verhaltensweisen haben die Gruppe bei der bisherigen Aufgabenlösung behindert?

Wie hat die Gruppe den bisherigen Prozeß der Problemlösung bewältigt?

Was würden die einzelnen Teammitglieder vorschlagen, um die Leistung der Gruppe zu verbessern?

Ergebnis der auf diese Weise angeregten Reflexionsprozesse muß es in erster Linie sein, bei den Teammitgliedern die bestehenden kognitiven wie auch sozial-interaktiven Konzepte der Zusammenarbeit i.S. höherer Gruppenkohäsion und Arbeitseffektivität $\mathrm{zu}$ verändern und dadurch adäquate Verhaltensänderungen $\mathrm{zu}$ initiieren, die wiederum nachhaltigen Einfluß auf die zweite Periode der Zusammenarbeit haben.

Diese Idee, an der Selbstreflexion der Gruppe anzusetzen, unterstützen auch die Ausführungen Moosmüllers (1997). Er geht davon aus, daß die Nachhaltigkeit der Resilienz und Leistungskraft einer bi-kulturellen Arbeitsgruppe am besten dadurch bewerkstelligt werden kann, wenn im Rahmen der psychologischen Begleitung gemischter Teams der interkulturelle Lernprozeß und der Erwerb 'interkultureller Handlungskompetenz' durch die Offenlegung des impliziten interkulturellen Wissens einer bi-kulturellen Gruppe angeregt, d.h. über die Bewußtmachung der in der Teamarbeit sich stetig ergebenden gemeinsamen interkulturellen Erfahrungen forciert wird; die Explikation und Kontrastierung vermeintlich faktischer Kulturunterschiede - wie sie bspw. durch die Präsentation und Diskussion der deutsch-tschechischen Kulturstandards erreicht würde (vgl. Novy \& Schroll-Machl, 2000) - wäre dieser Auffassung nach ein eher ungeeignetes Mittel der Teamentwicklung.

\section{Die Arbeit mit Stereotypen und die Beobachtung des Interaktionsverhaltens}

Insbesondere aufgrund der Relevanz der informellen Kontaktpflege (Novy \& Schroll-Machl, 2000; vgl. Eberhardt, 2000) zwischen und deutschen und tschechischen Kollegen bietet es sich an, sowohl die erste Intervention (Erstkontakt) als auch die zweite Intervention (Halbzeit der Projektarbeit) im Rahmen eines ein bis drei tätigen Work-Outs zu organisieren. Um besagte Selbstreflexionsprozesse in Gang zu setzen, müssen grundsätzlich die im Team virulenten Stereotype sowie das konkrete Interaktionsverhalten erhoben werden; 
es empfiehlt sich i.d.Z., auf computergestützte Unternehmensplanspiele, während deren Durchführung das Interaktionsverhalten videographiert wird, sowie auf daran anschließende halbstrukturierte Feedbackgespräche, in denen auf der einen Seite besagte Stereotype eruiert werden und auf der anderen Seite eine fundierte Rückmeldung über das konkrete Arbeitsverhalten erfolgt, zurückzugreifen; denn nach Badke-Schaub \& Frankenberger (1996) evozieren die hinter derartigen Planspielen befindlichen komplexen Problemlöseszenarien die selben Interaktionsverhaltensweisen, wie sie auch in realen Arbeitssituationen zu beobachten sind.

Befunde aus der empirischen Analyse der kulturellen Bedingtheit des Problemlöseverhaltens und der Zusammenarbeit (vgl. Eberhardt, 2000) können vom Trainer an dieser Stelle als 'didaktische Aufhänger bzw. Reflexionsanker" genutzt werden: sie sind für das einzelne Teammitglied Anstoß, die eigenen Vorstellungsmuster über das, was als prototypisch eigenkulturell (Autostereotyp), und das, was als prototypisch fremdkulturell erachtet wird (Heterostereotyp) zu explizieren, (vorerst wertfrei) zu reflektieren und im Laufe des Prozesses der Auseinandersetzung mit den Vorstellungen des anders kulturellen Partners ein erstes Verständnis für das systemische Bedingungsgefüge der interkulturellen Begegnungssituation zu entwickeln. Im Gegensatz zur Darstellung von Kulturstandards bietet dieser Ansatz, mit Stereotypen zu arbeiten, dem Teammitglied einen intensiveren Einblick in die Interaktionsdynamik/Intragruppendynamik der interkulturellen Begegnung, indem er a priori die interkulturellen Eingangserwartungen, die jedes Teammitglied in den Erstkontakt einbringt transparent und somit der Diskussion zugänglich macht; die angeregten Selbst- und Fremdreflexionen stellen damit den ersten Schritt für die Initiierung von Veränderungsprozessen dar, wie sie die Teamentwicklung fordert (vgl. Moosmüller, 1997). Die Schlußfolgerung, daß Kulturstandards aufgrund ihrer kontrastierenden und polarisierenden Wirkung demgegenüber ein eher ungeeignetes Medium zur Initiierung von Teambildungsprozessen darstellen, da sie oftmals und vorschnell als ,,[...] im Gegensatz zu Stereotypen - objektive Aussagen über Merkmale anderer Kulturen " betrachtet werden (Marada, 1997, S.46), wurde in Untersuchungen insbesondere von psychologisch geschulten Interviewpartnern bestätigt (Eberhardt, 2000). Diesbezüglich ist zu vermerken: Hofstede (1991; zit.n. Nehring, 1995) weißt der BR Deutschland einen leicht überdurchschnittlichen und der Tschechischen Republik einen extrem hohen Punktwert auf der Skala des Unisicherheitsvermeidungsindexes (UVI) zu; da Stereotype in der interkulturellen Begegnung immer die Funktion haben, Unsicherheit zu vermeiden, ist davon auszugehen, daß Stereotype gerade für die in der deutschtschechischen Begegnung ablaufenden Wahrnehmungs- und Attributionsprozesse eine herausragende Rolle spielen. Die Supervision und Begleitung einer deutsch-tschechischen Teamentwicklung ist deshalb gut 
beraten, die Bearbeitung von Auto- und Heterostereotypen sowie die dadurch angeregten Selbst- und Fremdreflexionen zu ihrem Gegenstand zu machen.

Es kommt ein weiterer Aspekt hinzu: die Darstellungsform kultureller Unterschiede im Rahmen einer Teamsitzung darf sich nicht einzig daran orientieren, bestehende Kontraste $\mathrm{zu}$ beschreiben, sondern muß vielmehr die Option der Veränderlichkeit und Veränderbarkeit kulturspezifischer Orientierungsmuster (auf national-kultureller Ebene aber auch auf der Ebene der Interkultur einer konkreten Arbeitsgruppe) ins Bewußtsein der Teammitglieder rücken (Moosmüller, 1997; vgl. auch Demorgon \& Molz, 1996). Wenn es Ziel einer bi-kulturellen Teamentwicklung ist, daß die Mitglieder die Wirksamkeit kultureller Einflüsse auf die Zusammenarbeit $\mathrm{zu}$ verstehen und i.S. 'interkultureller Handlungskompetenz' mit kultureller Vielfalt umzugehen lernen, dann empfiehlt es sich, den Teammitgliedern im Erstkontakt bewußt zu machen, daß zwar einerseits ,,[...] die 'Kulturbrille' [...] nicht abgelegt werden [kann, ... die Selbst- und Fremdwahrnehmung per definitionem immer] perspektivisch, [...] und 'Wirklichkeit' [daher immer ...] standpunkt- und situationsabhängig [...] “ ist (Moosmüller, 1997, S.290), daß aber andererseits das 'Erlebnis des Fremden' nicht einer konstanten Qualität der menschlichen Wahrnehmung entspricht, sondern vielmehr Ausdruck einer bestimmten Beziehungsweise zwischen Rezipient und Rezipiertem darstellt und somit wandelbar und beeinflußbar ist (vgl. Watzlawick, 1996). Da Auto- und Heterostereotype als intersubjektiv geteilte kognitive Strukturen zu betrachten sind und sie damit dem, der sich im Rahmen einer Teamentwicklung bewußt mit ihnen auseinandersetzt, von vornherein ihre 'Standpunkt- und Situationsabhängigkeit' vor Augen führen, eignen sie sich eher als - zumindest für den Moment als definitiv konstatierte - kulturelle Unterschiede für die Initiierung und Förderung einer Sensibilität gegenüber der in der interkulturellen Begegnung wirksamen Problematik der Fremd- und Selbstwahrnehmung. Dieser Ansatz taugt insbesondere dann, wenn sich eine der beiden Kulturen durch ein gewisses Maß an 'Konfliktvermeidung' auszeichnet; daß dies im Rahmen einer deutsch-tschechischen Teamentwicklung tendentiell der Fall ist, zeigen sowohl die Ergebnisse Eberhardts (2000) als auch die von Novy \& Schroll-Machl (2000).

Das zweite Instrument - die Erfassung des konkreten Interaktionsverhaltens mittels der Durchführung eines Planspieles ergänzt die pädagogische Funktion der Reflexion von Stereotypen wie folgt: Der Vergleich zwischen den Inhalten der Stereotype und dem realiter gezeigten Arbeitsverhalten kann stereotype Vorstellungen 'rechtfertigen' oder relativieren, korrigieren und helfen, über die Möglichkeit der Selbstbeobachtung die gegenseitige Bedingtheit aufeinander bezogener Verhaltensmuster im Rahmen einer interkulturellen Begegnungssituation $\mathrm{zu}$ erhellen. Die gemeinsame Analyse des Interaktionsverhaltens und insbesondere das fundierte Feedback des Trainers 
tragen dazu bei, ein Verständnis um das 'Warum?' der eigenen und der fremden Denke und Verhaltensgewohnheit zu entwickeln und zugleich die Reaktionen der (fremdkulturellen) Interaktionspartner verstehbar zu machen.

Über die Vergleiche zwischen Fremd-, Selbstwahrnehmung und objektiver Beobachtung lassen sich (subjektiv wie auch objektiv) eindeutige Aussagen über die für die zu erwartende Qualität der Zusammenarbeit maßgeblichen interkulturellen Einstellungs- und Verhaltensdispositionen machen. Auf dieser Grundlage können die jeweils brisanten Schwachstellen oder auch Leistungspotentiale der zu supervidierenden Gruppe eruiert werden und gezielt Übungen zur Überwindung von Defiziten bzw. zur Förderung von Stärken durchgeführt werden.

Eine Wiederholung dieses Prozederes im Rahmen der zweiten Intervention zur Halbzeit der Projektarbeit gibt Aufschluß über den Fortschritt bzw. die Entwicklung der Interkultur/Intragruppenkultur und eröffnet wie oben dargestellt nochmals die Möglichkeit, gezielt auf Veränderungsprozesse Einfluß zu nehmen (vgl. Gersick, 1988). Der vorgeschlagene Ansatz zweier aufeinander aufbauender Interventionsmaßnahmen - eine ganz zu Beginn des Kontaktes und die andere zur Halbzeit der Projektarbeit - kann somit als sensibles Instrument für die Supervision eines Teambildungsprozesses betrachtet werden.

Bei der Durchführung einer Interventionsmaßnahme muß des weiteren in Betracht gezogen werden, daß sich die Notwendigkeit einer Teamentwicklung immer vor einem konkreten politischen wie auch betriebswirtschaftlichen Hintergrund stellt; im Falle einer deutsch-tschechischen Teamentwicklung ist dieser Hintergrund der sicher noch einige Jahrzehnte andauernde gesellschaftlich-okönomische Transformationsproze $ß$ und der damit verbundene Transfer von technologischem und organisationswissenschaftlichem Know-how von West nach Ost. Damit ist jede interkulturelle Forschung und auch Personalentwicklungsmaßnahme, die sich mit der kulturellen Begegnung zwischen Ost und West auf der Ebene betriebswirtschaftlich relevanter Interaktions-, Kommunikations-, Entscheidungs- und Führungsproblematiken etc. beschäftigt, zwangsläufig mit dem Problem konfrontiert, eine Kultur als die dominante und die andere als die dominierte wahrnehmen zu müssen. Auch diese Tatsache ist ein Argument für die Zweckmäßigkeit der Bearbeitung von Auto- und Heterostereotypen im Rahmen der Teamentwicklung. Im Gegensatz zu den Schlußfolgerungen von Novy \& Schroll-Machl (2000) kann deshalb davon ausgegangen werden, die Reflexion eigen- und fremdkultureller Orientierungsmuster bewußt vor dem Hintergrund der Anforderungen moderner Betriebsführungsmethoden (wie Projektmanagement und Projektgruppenarbeit) stattfinden zu lassen und insbesondere im Rahmen der zweiten Intervention zur Halbzeit der Projektarbeit klare Wertungen einzelner Vor- und Nachteile im Interaktionsverhalten wie im Problemlösestil vorzunehmen. Denn einerseits zeigten die Ergebnisse von Expertenbefragungen (vgl. Eberhardt, 2000) 
eindeutig, daß die deutsche Vorstellung von der Systematik, Strukturiertheit und Akkuratesse bei der gemeinsamen Bearbeitung komplexer Probleme im Team einen gewissen Vorbildcharakter hat und sich tschechische Denk- und Handlungsmuster (in erster Linie aufgrund immer noch mangelnder Vertrautheit mit marktwirschaftlichen Methoden und Arbeitsweisen, nicht aufgrund überdauernder kultureller Orientierungsmuster) vielfach als eher 'unangepaßt und defizitär' darstellen; andererseits zeigten sie aber auch, daß - gerade was die enge Zusammenarbeit mehrerer Handlungssubjekte in einem bi-kulturellen Kontext betrifft - eindeutige Ergänzungspotentiale zwischen deutschen und tschechischen Orientierungsmustern i.S. der Steigerung der Gruppeneffektivität existieren.

Die in diesen Untersuchungen immer wieder thematisierte Bedeutung der Dominanz-Reaktanz-Dynamik für die Entwicklung interkultureller Konflikte läßt den Schluß zu, die Problematik der mangelnden Angepaßtheit bestimmter Verhaltensmuster im Rahmen einer Teamentwicklung bearbeiten zu müssen. Der Druck zur konstruktiven Auseinandersetzung über die eigene und die fremde Rolle im Transformationsprozeß sollte dabei dermaßen erhöht werden, daß die Beantwortung der damit zusammenhängenden Fragestellungen bzgl. 'kultureller Defizite' aber auch 'Vorzüge' sowie der kulturellen Identität für das einzelne Teammitglied unausweichlich wird (vgl. Barthold \& Eberhardt, 1999). Ziel einer Teamentwicklung muß es sein, daß die 'Orientierung an ökonomischen Notwendigkeiten zum eigenen wie auch gemeinsamen Vorteil' von beiden Seiten als maßgeblich anerkannt wird, und somit die Problematik der kulturellen Fremd- bzw. Selbstbestimmung (wie sie sich insbesondere für die tschechischen Interaktionspartner immer wieder stellt) durch die Entwicklung eines Bewußtseins über die Möglichkeiten einer gemeinsamen, identitätsstiftenden Erfolgsorientierung und Wertschöpfung gelöst wird:

Erfolg in der Zusammenarbeit ist ein maßgeblicher Faktor für die Entwicklung einer sozial stabilen, sich aus den unterschiedlichen kulturellen Ressourcen nährenden und damit zur kreativen Problemlösung beitragenden Interkultur. Was Sherif 1948 in seinem Jugendlagerexperiment gemeinhin feststellte, gilt im Besonderen für die 'deutsch-tschechische Gruppe': gemeinsam erbrachte Leistung fördert die Gruppenkohärenz sowie die Qualität der Zusammenarbeit. Im Hinblick auf die Systematik einer konkreten Interventionsmaßnahme kann deswegen festgehalten werden, $d a ß$ es in einer deutsch-tschechischen Teamentwicklung immer darauf ankommt, Strukturen zu schaffen, die es erlauben, Erfolg als gemeinsamen Erfolg wahrzunehmen.

\section{Kulturkontraste als Potentiale einer höheren Gruppeneffektivität}

Um ein Bewußtsein für die Möglichkeit zu schaffen, im Rahmen einer Arbeitsgruppe gemeinsam erfolgreich sein zu können, ist es nötig die Schwächen und Stärken sowie die vielseitigen Ergänzungspotentiale zwischen 
zwei verschiedenen Orientierungssystemen zu kennen. Aufbauend auf den Untersuchungsergebnissen Eberhardts (2000), welche die kulturelle Bedingtheit von Handlungsregulationen im Kontext deutsch-tschechischer Zusammenarbeit beschreiben, wurde deshalb ein Profil von Kulturkontrasten erarbeitet. Die Kenntnis der in diesen Kontrasten implizierten Ergänzungs- und Anregungspotentiale kann helfen, die Gruppenleistung und Arbeitszufriedenheit in bi-kulturell zusammengesetzten Projektgruppen $\mathrm{zu}$ steigern. Die Orientierungsmuster und Handlungstendenzen sind in Anlehnung an die von Demorgon \& Molz (1996) vorgeschlagene Systematik der 'adaptiven Gegensätze' kontrastiv dargestellt, nach Inhalts-, Prozeß- und sozio-emotionalen Aspekten der Zusammenarbeit gegliedert und jeweils durch die im Rahmen von Expertenbefragungen berichteten Vor- und Nachteile der betreffenden Ausprägungen ergänzt.

Die Darstellung dieser 'Tendenzen', die in der deutsch-tschechischen Teamarbeit aufeinandertreffen (können), hilft, der Frage nachzuspüren, inwieweit es möglich ist, Unterscheide im kulturbedingten Interaktions- und Problemlöseverhalten gleichwertig $\mathrm{zu}$ berücksichtigen, so daß sie die 'Freiheitsgrade' des Handlungsrepertoires in der Gruppe erweitern, oder diese Unterschiede zu einem innovativen, gruppeneigenen Stil zu integrieren, der die jeweiligen Vorteile maximiert und die Nachteile vermeidet 


\title{
A. Inhaltsdimensionen
}

\section{Deutsch}

Vor- und

Nachteile

+ Intrinsische

Aufgaben-

motivation

- mangelnde

Sensitivität für

Andersartig-

keiten

+ Einblick in

Systemregeln

-Versachlichung

komplexer

Probleme,

Reduktionismus,

- "Verzetteln"

\author{
Extreme
Sachorientierung;
Betonung der
Aufgabeninhalte
}

Fokus

\section{Problemlösestrategie}

Ausgeprägte

Erkenntnis-

orientierung:

Komplexe Analyse von

Wirkungs-

zusammenhängen

(kausale

Situationsanalyse);

Stringente, umfassende

Datenhaltung; kritische

Antizipation von

Problemaspekten;

auftauchende

Problemstellungen bewirken

grundsätzliche

Problemanalysen;

Strategie: proaktiv,

antizipierend,

konzeptualisierend
Tschechisch

Vor- und

Nachteile

\begin{tabular}{l|l|} 
Situationsbedingte & + Achten auf \\
Fokussierung auf & positive \\
Aufgabeninhalte; & Gruppen- \\
Bemühen um & dynamik \\
Ausgleich zwischen & -mangelnde \\
Sachaspekten und & Internalisierung \\
Gruppendynamik & von Zielvorstel- \\
& lungen bedingt \\
& Zwang zur \\
& extrinsischen \\
& Aufgaben- \\
& motivation \\
\hline
\end{tabular}




\section{B. Prozeßdimensionen}

\section{Strukturierung}

\begin{tabular}{|l|}
+ Kosten- und \\
Zeitersparnis bei \\
routinisierten \\
Handlungen \\
+ Gründlichkeit \\
-mangelnde \\
Flexibilität \\
\end{tabular}

+ breit angelegte, rationale

Prüfung von Argumenten $+$

Sachdienlichkeit von Entscheidungen

- mangelndes Gespür für die herrschende Meinung

-Rationalisierung von egoistischen Motiven

-Unnachgiebigkeit bei 'Meinungsfragen"
Ausgeprägte Prozeßorientierung: Handlungsplanung und Strategieplanung; Zieldefinitionen dienen der Orientierung; monochrone Handlungsausführung; Schematisierung einzelner

Handlungsschritte; sachlich begründete Aufgabenteilung und Übernahme von Einzelverantwortung

\section{Entscheidungsfindung}

Konsens durch Überzeugung;

Schätzen von

Eigeninitiative;

uniforme

Entscheidungsfindung;

Durchsetzung von

Sachzwängen;

Auseinandersetzung mittels des argumentativen Diskurses
Wenig systematisches

Vorgehen:

Zielreflexionen dienen der groben

Eingrenzung des

Handlungsspielraums;

polychrone

Problembearbeitung;

Verteilung der

Ressourcen
+Improvisation; schnelle Umstellung auf sich verändernde Gegebenheiten

- mangelnde

Prozeßtransparenz und Kalkulierbarkeit
+ Kooperatives Klima

+Zeitersparnis in Diskussionen bei klaren hirarchischen Verhältnissen

Einholen von Rückversicherungen

+ Identifikation durch 'gemeinsam' getroffene Entscheidungen

Reibungsverluste durch subjektive Anstöße -Unreflektierte Übernahme suboptimaler Vorschläge -Teilnahmslosigkeit bei okroyierten Beschlüssen 
+ Reduktion von Unsicherheit und Risiko

-Handlungsblockierung durch Perfektionismus und mangelnde Kompromißbereitschaft

\section{Verbindlichkeit von Entscheidungen}

\begin{tabular}{|c|c|c|}
\hline $\begin{array}{r}\text { Sachorientierung; } \\
\text { Betrachtung der } \\
\text { 'gefundenen Lösung' } \\
\text { als einzig plausibler } \\
\text { Weg }\end{array}$ & $\begin{array}{l}\text { Zulassen provi- } \\
\text { sorischer Entschei- } \\
\text { dungen; } \\
\text { häufigere Revisionen; } \\
\text { hohe Verände- } \\
\text { rungsbereitschaft }\end{array}$ & $\begin{array}{l}\text { + Produktion } \\
\text { akzeptabler } \\
\text { Übergangs- } \\
\text { lösungen } \\
\text { + Flexible } \\
\text { Anpassung } \\
\\
\text {-Verhaften in } \\
\text { suboptimalen } \\
\text { Lösungs- } \\
\text { ansätzen } \\
\text {-mangelnde } \\
\text { Verbindlichkeit } \\
\text { von Absprachen }\end{array}$ \\
\hline
\end{tabular}




\section{Sozio-emotionale Dimensionen}

\section{Kommunikationsstil}

\begin{tabular}{|l|}
\hline + Transparenz, \\
Klarheit \\
-Distanz, wenig \\
persönlich \\
\\
\hline
\end{tabular}

+ Offene Klärung von Störungen

- Beziehungsverlust bei mangelnder Trennung zwischen 'Sache und Person'
Expliziter, direkter

Code;

Wenig lebhafte

Interaktion;

Konfrontative

Unterbrechungen

(Oppositionsformat);

Verpflichtung zur

Darlegung der eigenen

Handungsperspektive

\section{Kritikäußerung}

\begin{abstract}
Direkte Äußerung sachlicher wie persönlicher Kritik
\end{abstract}

Impliziter, indirekter

Code (,Sprache als

Spiel");

Lebhafte, humorvolle Interaktion;

Affirmative

Unterbrechungen;

Schaffung

'augenzwinkernder

Verbundenheit";

Aktive Exploration der Handlungsperspektive des Partners
Schnelligkeit und Effizienz der Kommunikation im 'vertrauten Rahmen'

+ 'vornehme' Zurückhaltung, Vorsicht gegenüber Fremdem

-dauerhafte

Skepsis

- 'Mißbrauch' der Sprache zur InGroup-OutGroupMarkierung
Vermeidung oder indirekte Äußerung persönlicher Kritik
+ Rücksichtsvoller Umgang miteinander

-Totschweigen dringender Probleme; Spannungsaufbau und plötzliche Entladung im Konflikt 


\section{Konfliktbewältigung}

\begin{tabular}{|l|}
\hline+ Transparenz; \\
konstruktive \\
Bearbeitung \\
schafft \\
Erweiterung der \\
Konfliktlösungs- \\
strategien \\
-Dominanz; \\
einseitiges \\
Durchsetzen des \\
'Stärkeren' \\
\hline
\end{tabular}

+Offenlegung von Verbesserungspotentialen

+ Transferlernen

-Nörgelei und Skeptizismus

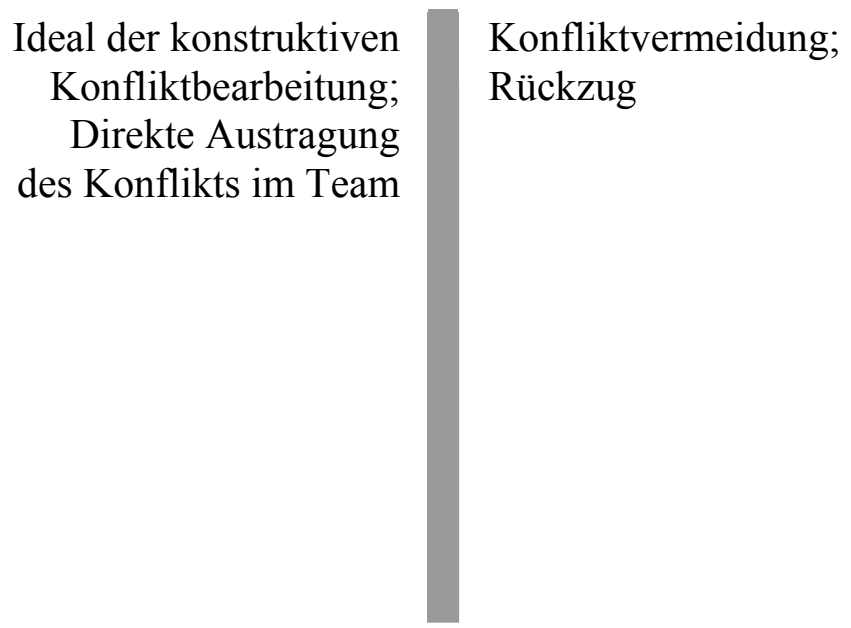

\section{Arbeits- \& Leistungsbewertung}

Individuelle

Zuweisung von Erfolg und Mißerfolg;

Bemühen um sachliche

Evaluierung;

Kenntnisnahme

von Mißerfolgen

und Fehlern;

Explikation von

Mißständen zum

Zwecke einer

nachhaltigen

Lösungsfindung und

des Transfers auf

künftige Situationen
+ Wahrung der Gruppenharmonie

-Reaktanz; Trotz; stiller Widerstand gegen empfundene Fremdbestimmung

+ Gruppenkohäsion durch das Erleben gemeinsamer Erfolge

Ergebnissen;

'Schweigen' bei negativen Ergebnissen

\section{-Negieren und} Ignorieren von Fehlleistungen

-Scheu vor kritischer Bewertung 


\section{Beziehungskonstitution}

\begin{tabular}{|l|}
\hline + Schnelligkeit, \\
Professionalität \\
der Aufgaben- \\
bewältigung; \\
ausgeprägte \\
Leistungs- \\
orientierung; \\
Wille zur \\
'Pflichterfüllung \\
' \\
-Anonymität, \\
mangelnde \\
Rücksichtnahme \\
auf persönliche \\
Belange \\
\hline
\end{tabular}

Ernsthaftes, sachorientiertes, analytisches Herangehen an die Aufgabe; Die Qualität der Beziehungsebene folgt dem Ergebnis der gemeinsamen Sacharbeit
Anfängliche Konzentration auf die Beziehungsebene; Auf der Grundlage positiver Gruppenbeziehungen kann erfolgreiche Sacharbeit geleistet werden
+ Schaffen einer
'Vertrauens-
atmosphäre';
nachhaltige
Hilfsbereitschaft
bei Sympathie
-Langwierigkeit

\section{Reaktion auf Frustration}

\begin{tabular}{|l|}
\hline+ Eigener \\
Spannungsabbau \\
+nachhaltige \\
Arbeit an der \\
Problembewälti \\
gung \\
-Persönliche \\
Verletzungen \\
-Verbissenheit \\
\end{tabular}

+ Gesicht wahren; ausgeprägte

Duldsamkeit Rechtfertigung eigener Fehler und argumentative Begründung des Versagens; emotionale Reaktionen und internale Problembearbeitung auf nachweisliche Fehlleistungen
Rückzug oder impunitive Äußerungen;

Sachlichkeit als Indikator für Streß und Frustration; 'Rückzug ins Sachliche'
-Fatalismus:

"Ertragen" von Demütigungen und Niederlagen; Selbstbild vom „Gepeinigten“

\section{Literatur}

Badke-Schaub, P., Frankenberger, E. (1996). Von den Daten zum Modell. Eine empirische Untersuchung von Gruppenarbeit in der Konstruktionspraxis. Memorandum Nr. 14, Lehrstuhl Psychologie II der Otto-Friedrich-Universität Bamberg.

Barthold, H.-P. \& Eberhardt, C. (1999). Coaching - Concerning the Cultural Differences. Zum Problem der Übertragung von Coaching-Ansätzen auf fremdkulturelle Anwendungsfelder. Unveröfftl. Arbeitspaper im Rahmen der Einführung von Coachingmaßnahmen durch die VW-Coaching. SKODA Auto a.s. Mlada Boleslav, Leitung der Fort- und Weiterbildung ZV.

Demorgon, J. \& Molz, M. (1996). Bedingungen und Auswirkungen der Analyse von Kultur(en) und interkulturellen Interaktionen. In Thomas A. (Hrsg.), Psychologie interkulturellen Handelns (S. 43 -86). Göttingen: Hogrefe. 
Eberhardt, C.J. (2000). Zur kulturellen Bedingtheit von Handlungsregulationen im Kontext deutsch-tschechischer Zusammenarbeit. Unveröfftl. Diplomarbeit, Universität Regensburg.

Francis, D. \&Young, D. (1989). Mehr Erfolg im Team. Hamburg.

Gersick, C. J. G. (1988). Time and transition in work teams: Toward a new model of group development. Academy of Management Journal, 31, pp. 9-41.

Gersick, C. J. G. \& Hackman, J. R. (1990). Habitual routines in task-performing groups. Organizational Behavior and Human Decision Processes, 47, pp. 65-97.

Hofstede, G. (1991). Interkulturelle Zusammenarbeit. Verlag: Gabler.

Marada, R. (1997). Kulturstandards und postkommunistische Transformation. Das Beispiel Tschechien (S.45-62). In Höhne, S. \& Nekula, M. (Hrsg.), Sprache, Wirtschaft, Kultur. München: iudicium.

Moosmüller, A. (1997). Kommunikationsprobleme in amerikanisch-japanisch-deutschen Teams: Kulturelle Synergie durch interkulturelles Training?. ZfP Zeitschrift für Personalforschung, 1997, 3, S.282-297.

Novy, I. \& Schroll-Machl, S. (2000). Perfekt geplant oder genial improvisiert? Kulturunterschiede in der deutsch-tschechischen Zusammenarbeit. München und Mering: Rainer Hampp Verlag.

Nehring, H. (1995). Working in the Czech Republic: Management and Communication Styles. In: Bozena Máchova/Slava Kubátová (Hrsg.), Sietar Europa 1995, Proceedings. Uniqueness in Unity. The Significance of Cultural Identity in European Cooperation (S.250-259). Praha.

Simon, P. (2000, in Druck). Entwicklung eines Modells der Gruppeneffektivität und eines Analyseinstruments zur Erfassung des Leistungspotentials von Arbeitsgruppen. Dissertation an der Universität Regensburg.

Tuckman, B. W. (1965). Development sequence in small companies. Group and Organizational Studies, 2, pp. 419-427.

Watzlawick, P. (1996). Menschliche Kommunikation. Verlag Hans Huber. 